\title{
Doped nanoparticles for optoelectronics applications
}

\author{
M. Godlewski ${ }^{*, * *}$, E. Wolska**, and S. Yatsunenko* \\ "Institute of Physics Polish Academy of Sciences, Al. Lotnikow 32/46, 02-668 Warsaw, Poland \\ E-mail: godlew@ifpan.edu.p \\ ${ }^{* *}$ Department of Mathematics and Natural Sciences, College of Sciences, \\ Cardinal S. Wyszyński University, Warsaw, Poland
}

A. Opalińska, J. Fidelus, and W. Łojkowski

Institute of High Pressure PAN «Unipress», Sokołowska 29/37, Warsaw, Poland

\section{Zalewska and A. Kłonkowski}

Faculty of Chemistry, University of Gdańsk, Sobieskiego 18, 80-952 Gdańsk, Poland

\section{Kuritsyn}

Institute for Physics of Microstructures Russian Academy of Science, GSP-105, Nizhny Novgorod, 603950 Russia

Received July 8, 2008

\begin{abstract}
Nanoparticles of wide band gap materials doped with transition metal ions or rare earth ions are intensively studied for their possible applications in a new generation of light sources for an overhead illumination. In this work we discuss mechanisms of emission enhancement in nanoparticles doped with rare earth or/and transition metal ions. Arguments are presented that phosphors of nanosize may emit light more efficiently and thus be applied in practical optoelectronic devices.
\end{abstract}

PACS: $81.07 . W x$ Nanopowders;

78.47.-p Spectroscopy of solid state dynamics;

78.55.-m Photoluminescence, properties and materials;

78.55.Hx Other solid inorganic materials.

Keywords: excitation processes in nanoparticles, photon cascade processes.

\section{Introduction - why we need new light sources for overhead illumination?}

At present, incandescent and fluorescence lamps (also in the form of compact fluorescence lamps (CFLs)) are used for an overhead illumination. The widely used incandescent lamps with tungsten filament (about 9 billion pieces is used in the world) are very inefficient. The efficiency depends slightly on their power (for example, it is about $1.9 \%$ for $40 \mathrm{~W}$ lamp and about $2.1 \%$ for $60 \mathrm{~W}$ lamp and $2.6 \%$ for $100 \mathrm{~W}$ lamp) and temperature (efficiency reaches about 5\% for high-temperature tungsten incandescent lamps), but in all these cases it is still very low as compared to alternative light sources. This situation is not changed by the fact that lamp's efficiency can be slightly improved by coating of an inner part of glass bulb with highly conductive transparent (for visible light) oxide reflecting back an infrared radiation.
For ideal white light sources one expects more than $35 \%$ efficiency. Such efficiency can not be reached for lamps based on a concept of black body radiation. Thus, what we need is a replacement of incandescent lamps with lamps emitting monochromatic radiation. This replacement will result in a huge money (more than 80 billion USD per year) and energy saving, resulting also in a reduced by hundreds of millions of tones emission of $\mathrm{CO}_{2}$ gas to the atmosphere and lower electricity consumption [1].

As replacement we can use either CFLs or so-called white light emitting diodes (w-LEDs). The latter are GaN-based quantum well (QW) devices, in which primary blue emission of LED is mixed with a phosphor(s) (one or two) emission (see [2] and references given there). These are so-called hybrid diodes, since in these devices a phosphor emission is excited by diode emission, 
and white color impression is obtained by mixing of a primary LED radiation and LED-excited phosphor emission.

In the first generation of commercialized w-LEDs $400 \mathrm{~nm}$ blue/violet emission from InGaN quantum wells was mixed with a yellow emission of YAG:Ce phosphor to achieve an impression of a white color $[3,4]$. This concept (hybrid LED) is still used in recent more efficient w-LEDs. One can expect an improved efficiency not only when primary LED emission will be optimized, but also if more efficient phosphors will be developed, which means that also in this case we will benefit from introduction of efficient light conversion phosphors.

\section{Experimental setups}

The present study is mostly based on the results of photoluminescence (PL), PL excitation (PLE) and PL kinetics investigations of different nanoparticles and quantum structures. Details on sample studied and experimental set up used can be found elsewhere [5-12].

\section{Discussion - how to improve luminophors?}

There are several alternative ways to improve efficiency of presently used luminophors. These can be divided to a few steps. Efficiency will be improved by: a) more efficient excitation; b) more efficient host-to-impurity energy transfer; c) more efficient radiative recombination; and d) less efficient channels of nonradiative recombination. In this work we shortly described these steps, which relate to a small size of powders studied. Benefits of introducing nanopowders will be indicated.

\subsection{Enhanced rate of excitation process in nanoparticles}

Presently used fluorescent phosphors often show high quantum efficiency of the light emission. However, their application is limited by relatively narrow excitation regions. Phosphors were optimized to work with a given device, e.g., as UV-visible light converters in fluorescence lamps. Thus, phosphors optimized for the use in CFLs were not suitable for LED applications.

To get spectrally narrow emissions phosphors are doped with either transition metal (TM) or rare earth (RE) ions. In the latter case this introduces a serious limitation. $4 f-4 f$ intra-shell excitation is fairly inefficient (parity forbidden process) and thus $4 f-5 d$, charge transfer or excitonic mechanism of excitation must be used [13] This however, means that hosts with large band gap must be used to allow such transitions. This limits quantum efficiency of the excitation (see discussion below) and limits available materials.

Moreover, intra-shell $3 d-3 d$ or $4 f-4 f$ transitions are often spin forbidden, which further limits rate of radiative recombination. For example, the ${ }^{4} T_{1}$ to ${ }^{6} A_{1}$ intra-shell transition of $\mathrm{Mn}^{2+}$ ions is a parity and spin (transition between spin quartet and sextet) forbidden process. Thus, this transition is fairly inefficient in bulk samples, especially in sulfides and selenides [5-12].

Most of these limitations can be avoided by the use of nanoparticles as host lattices. These nanocrystals, when of nm sizes, have properties dictated by quantum confinement effects, but most of all sizes small enough to allow light penetration even if photons with above band gap energy are used. In Fig. 1 we show PL excitation (PLE) spectra measured by us for terbium doped $\mathrm{ZnS}$ and $\mathrm{ZrO}_{2}$ nanoparticles (see [12] for the details on sample techno$\operatorname{logy}$ and properties). In both cases efficient $4 f-4 f$ intra-shell PL of $\mathrm{Tb}^{3+}$ is induced upon $4 f-5 d$ excitation of $\mathrm{Tb}^{3+}$. In both host materials this is the dominant PL excitation band. A surprising result is observation of this PLE band, despite that its energy is above band gap energy.

Another nanosize-related effect was reported by us recently [12]. We demonstrated that the ${ }^{4} T_{1}$ to ${ }^{6} A_{1}$ intra-shell PL of $\mathrm{Mn}^{2+}$ ions in $\mathrm{ZnS}$ nanoparticles can be efficiently pumped under band-to-band excitation, which was not observed for bulk $\mathrm{ZnMnS}$ (see Fig. 2). In the process described by us in the Ref. 12 we observed that band-to-band excitation of host $\mathrm{ZnS}$ nanoparticles is followed by efficient host-to-impurity energy transfer. We concluded that enhanced host to $\mathrm{Mn}^{2+}$ energy pumping is related to confinement enhanced $\mathrm{Mn}$-free carrier interactions. We also demonstrated that the above-mentioned interaction affects rates of intra-shell transitions as long as photoexcited free carriers coexist with excited $\mathrm{Mn}^{2+}$ ions (see [12] and references given there).

For RE ions one of possible ways of their excitation is energy transfer from other center. Such transfer requires energy overlap between energy donor and energy acceptor (RE ion in this case). Once we noticed highly efficient

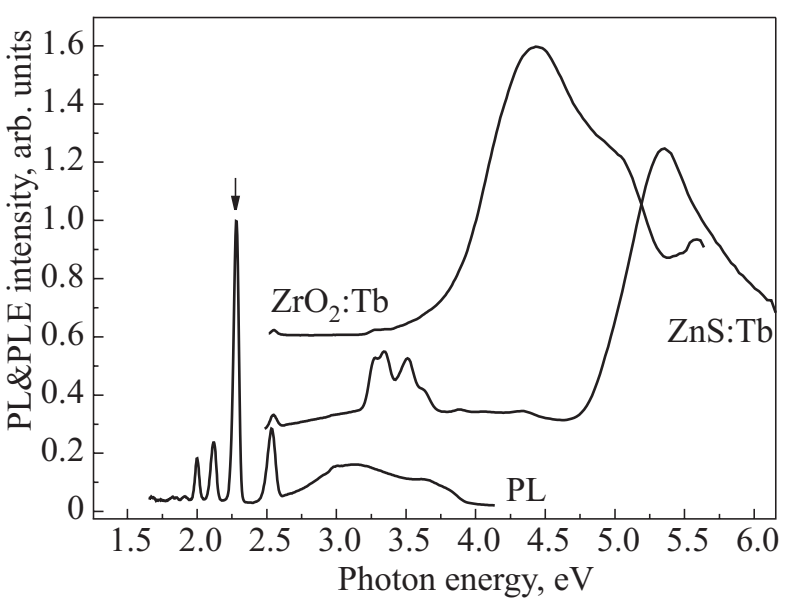

Fig. 1. PL excitation spectrum of $\mathrm{Tb}^{3+} \mathrm{PL}$ in $\mathrm{ZnS}$ and $\mathrm{ZrO}_{2}$ nanoparticles. 


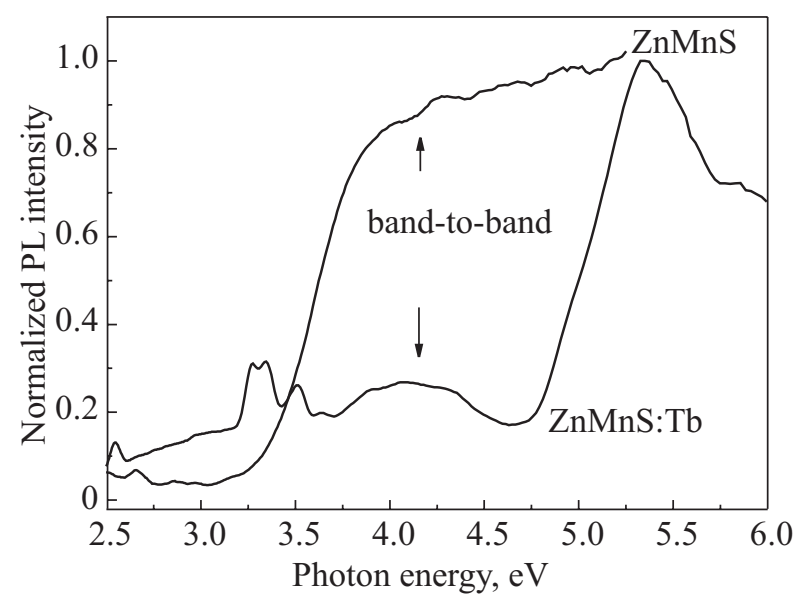

Fig. 2. PL excitation spectra of $\mathrm{Mn}^{2+}$ and $\mathrm{Tb}^{3+} \mathrm{PL}$ in $\mathrm{ZnMnS}$ and $\mathrm{ZMnS}: \mathrm{Tb}$ nanoparticles.

host-to- $\mathrm{Mn}^{2+}$ energy transfer in $\mathrm{ZnS}$ nanoparticles, we proposed stimulation of $\mathrm{Tb}^{3+} \mathrm{PL}$ by codoping of $\mathrm{ZnS}: \mathrm{Tb}$ nanoparticles with manganese ions. In Fig. 2 we show PLE band of $\mathrm{Tb}^{3+} 4 f-4 f$ emission observed in $\mathrm{ZnS}$ nanoparticles codoped with Mn ions. A new broad PLE band (indicated with arrow) is observed, which is due to the band-to-band host excitation. Apparently, in codoped samples host-to-RE energy transfer proceeds via host-to-Mn transfer, followed by Mn-to-RE transfer. In the process $\mathrm{Mn}^{2+}$ intra-shell PL is deactivated (see [12] for further details). Even though the new excitation mechanism is still less efficient than the $4 f-5 d$ one, it still opens a new possibility of tuning of the excitation energy due to quantum confinement effects. By selecting size of nanoparticles we can shift the energy of their fundamental absorption band and thus tune RE excitation energy, when using the above-described PLE process.

Another situation we show in Fig. 3. There we compare efficiency of host-related and $4 \mathrm{f}-5 d$ excitation processes of $\mathrm{Pr}^{3+}$ ions in $\mathrm{ZrO}_{2}$ lattice. There the host excitation of $\mathrm{RE}$

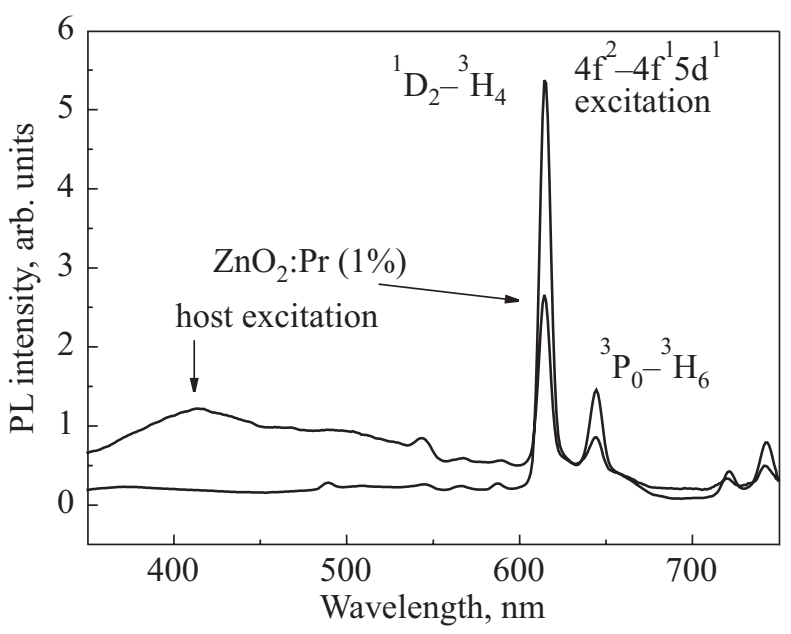

Fig. 3. PL emission of $\mathrm{Pr}^{3+}$ in $\mathrm{ZrO}_{2}$ nanoparticles observed at room temperature under host and $4 f-5 d$ excitation. emission was observed due to a spectral overlap of host emission and $\mathrm{Pr}^{3+}$ emission. However, in this case excitation of the intra-shell PL is rather ineffective.

\subsection{Enhanced rate of radiative decay processes in nanoparticles}

In a very inspiring work Bhargava and co-workers [14] reported that in $\mathrm{ZnMnS}$ nanocrystals the observed PL decay time of the ${ }^{4} T_{1}$ to ${ }^{6} A_{1}$ transition of $\mathrm{Mn}^{2+}$ was shorter by 5 orders in magnitude. 3.7 and 20.5 ns PL decay time was observed instead of ms one [14]. Such lifetime shortening was observed together with an increase in emission efficiency, i.e., the effect could not be related to enhanced rate of a nonradiative decay [14]. Even though the proposed model of quantum confined atom turned out to be wrong, our following works confirmed the appearance of short components of the PL decay in TM/RE doped nanoparticles [5-13]. The most important result of these studies was observation of the co-existence of fast and slow components of the PL decay in all the samples studied, with the fast component of the PL decay being very enhanced in nanocrystals. The latter result was in agreement with the results reported by Bhargava and co-workers [14].

We also accounted for the co-existence of various PL decay components. We found that the fast PL decay is only seen if free carriers are co-excited. Once free carriers decay, are trapped etc... the mechanism of the ${ }^{4} T_{1}$ to ${ }^{6} A_{1}$ $\mathrm{PL}$ enhancement is deactivated and the slow PL decay of the $\mathrm{Mn}^{2+}$ intra-shell PL should be observed [5-13]. This however, turned out not to be the case. In all systems studied by us a multicomponent PL decay was observed.

Such multicomponent, nonexponential PL decay of TM or RE emission can often be related to the competition of nonradiative recombination transitions, as was reported recently [15]. We reported however (see [16] for the details) that a similar PL decay is also observed for high quality CdMnTe quantum dots samples grown by molecular beam epitaxy, in which radiative recombination processes dominated.

Possible explanation of the multicomponent PL decay (first, after an intensive excitation, PL decays fast, which then turns to a slow decay expected for parity forbidden $3 d-3 d$ or $4 f-4 f$ transitions) comes from the old work of Dicke (from 1954) [17]. In accordance to the results of this work, a spontaneous emission of diluted nanoparticles or quantum dots must include their interaction with a radiation field, resulting in a cooperative radiation-a super-radiance [17]. The super-radiance is also expected for a system of self-assembled quantum dots [18].

Indirect evidence for super-radiance in RE doped nanoparticles was based on confocal microscopy investigations of PL kinetics of $\mathrm{Gd}_{2} \mathrm{O}_{3}$ :Sm nanoparticles diluted in a glycol [16]. We reported the appearance of a fast com- 
ponent (ns) of the $\mathrm{Sm}^{3+} \mathrm{PL}$ decay. We also reported that the PL enhancement is observed together with the appearance of fast nanoseconds component of the PL decay.

Super-radiance is only expected at initial stages of the PL decay. When concentration of excited ions drops their emission decouples and «normal» PL decay should be observed. The mechanism should be very enhanced in the case of emitters (TM or RE ions, other dopants, quantum dots, ...) emitting at the resonant energy. Thus, heavily excited system of RE doped nanoparticles is an ideal system to evidence such process. The problem with the process identification relates to the fact that a nonradiative decay often should show a similar effect on the PL decay, i.e., the fast decay should be observed at first, followed by a «normal» slow decay, due to parity forbidden $3 d-3 d$ or $4 f-4 f$ transitions. Nonradiative decay is enhanced by an energy migration via excited states of TM or RE ions. We used this fact to distinguish between nonradiative and enhanced radiative (likely due to the super-radiance) decay. In Fig. 4 we compare an ultrafast component of the PL decay observed for $\mathrm{Eu}^{3+}$ doped nanoparticles with two different RE impurity content (5 and 15\%). The experiment was performed at the same excitation condition. In Fig. 4 one can see that the ultrafast components of the PL decay are identical, within the experimental accuracy, for nanoparticles with different concentration of the Eu ions. This was not expected for the dominant nonradiative channel of the recombination, but such dependence is likely to occur for the dominant super-radiance mechanism.

\subsection{Size dependence}

The above described processes indicate that the RE emission can be enhanced in nanoparticles. First of all due to the fact that host-to-ion energy transfer is enhanced (PL will be more efficient, primary due to more efficient

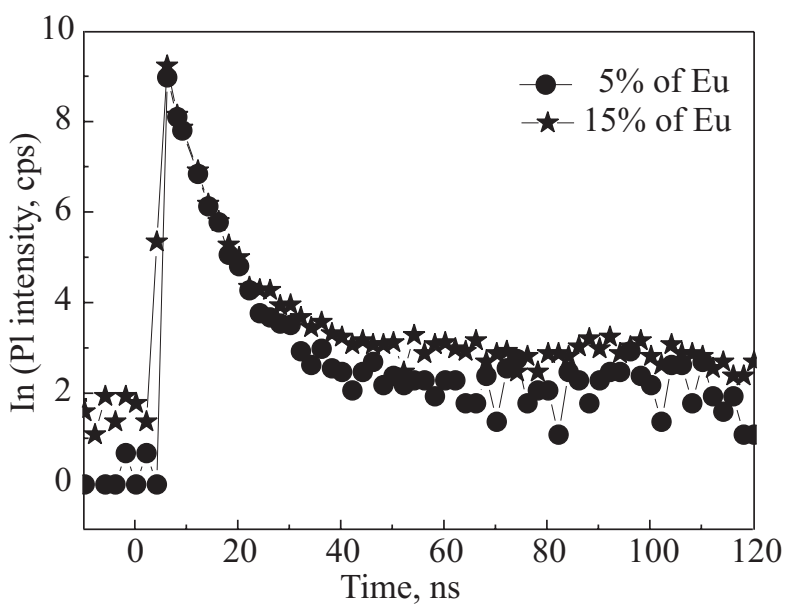

Fig. 4. The ultrafast component of $2 \mathrm{~K}$ PL decay of $\mathrm{Eu}^{3+} 4 f-4 f$ PL measured for samples containing two different Eu fractions. energy pumping). The following $4 f-4 f$ deexcitation can also be enhanced by the super-radiance mechanism of the PL decay. The latter mechanism is also expected in bulk samples and thin films, so the open question remains if nanosize is here of importance. In particular if the efficient mechanism evidenced by us for $\mathrm{Mn}^{2+}$ ions (due to strong $\mathrm{Mn}$-free carriers interactions in $\mathrm{ZnS}$ nanoparticles, see Refs. 5-12 for the details) is expected to be less efficient for RE ions, due to screening of the internal $4 f$ shell by external $5 s$ and $5 p$ electrons. This is why we looked for further evidence that nanosize of particles is important.

To verify this fact we measured influence of nanoparticles size on PL efficiency of two RE ions in the same host. $\mathrm{Pr}^{3+}$ and $\mathrm{Tb}^{3+}$ doped $\mathrm{ZrO}_{2}$ nanoparticles were studied, with their size regulated by a post-growth annealing. By annealing in the temperature range of room temperature $-900{ }^{\circ} \mathrm{C}$ we could vary size of $\mathrm{ZrO}_{2}$ particles between about $10 \mathrm{~nm}$ (in as-grown nanoparticles) and $100 \mathrm{~nm}$ (in nanoparticles annealed at $900{ }^{\circ} \mathrm{C}$ ). Details on this procedure will be given elsewhere [19].

Figure 5 shows an interlink between an integrated PL emission of $\mathrm{Pr}^{3+}$ ions and annealing temperature (size of nanoparticles). The decrease of the PL intensity is relatively large in the largest nanoparticles - the PL intensity decreases by more than 4 times, mostly due to decrease in intensity of the red $\left({ }^{1} D_{2}-{ }^{3} H_{4}\right)$ component of the $4 f-4 f$ emission. Similar dependence we also observed for $\mathrm{Tb}^{3+}$ PL in the same host material [20].

\subsection{Cascade transitions (photon cutting)}

At first view phosphors used in fluorescence lamps very efficiently convert UV photon emitted by $\mathrm{Hg}$ vapours to a visible light. The reported very high quantum efficiency of the process relates to the fact that one UV photon emitted by $\mathrm{Hg}$ vapours is converted to one visible

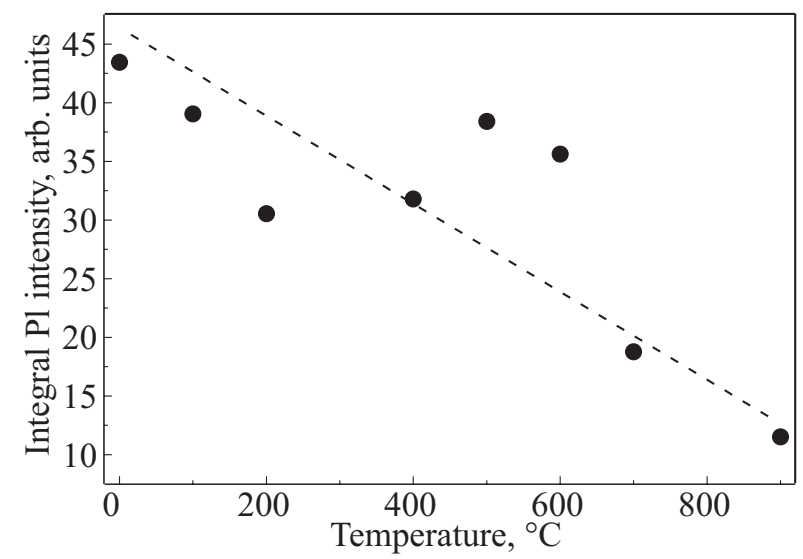

Fig. 5. Dependence of the integrated PL intensity of $\operatorname{Pr}^{3+}$ ions in $\mathrm{ZrO}_{2}$ measured for the sample with $1 \%$ Pr fraction for as grown sample (of $10 \mathrm{~nm}$ size) and annealed at temperatures up to $900{ }^{\circ} \mathrm{C}$ (nanoparticles size increases to $100 \mathrm{~nm}$ ). 
photon. Only if counted on this way the efficiency of a light conversion approaches $100 \%-1$ UV photon excites 1 visible photon. The efficiency is however much lower if we calculate photons energy. It was pointed out that this efficiency can be improved if one UV photon emitted by $\mathrm{Hg}$ will excite two (or even more) visible photons in a cascade process. Such situation was found for $\mathrm{Pr}^{3+}$ ions in several host materials [21-26].

Energy structure of $\mathrm{Pr}^{3+}$ consists of ${ }^{3} \mathrm{H},{ }^{3} \mathrm{~F},{ }^{1} \mathrm{D},{ }^{1} \mathrm{G},{ }^{3} \mathrm{P}$, ${ }^{1} I,{ }^{1} S$ multiplets split by the spin-orbit interaction to ${ }^{3} \mathrm{H}_{4}{ }^{3} \mathrm{H}_{5}{ }^{3} \mathrm{H}_{6},{ }^{3} \mathrm{~F}_{2}{ }^{3} \mathrm{~F}_{3}{ }^{3} \mathrm{~F}_{4},{ }^{1} G_{4},{ }^{1} D_{2},{ }^{3} P_{0}{ }^{3} P_{1}{ }^{3} P_{2},{ }^{1} I_{6}$ and ${ }^{1} S_{0}$ states [27], as shown in Fig. 6 . In the photon cutting (photon cascade) process reported in the reference [21] the efficient $4 f^{2}$ to $4 f^{1} 5 d^{1}$ excitation is followed by a carrier trapping via the highest lying $4 f$ excited state ${ }^{1} \mathrm{~S}_{0}$ state and a cascade process, in which two or more visible photons are emitted: ${ }^{1} S_{0^{-}}{ }^{3} P_{1},{ }^{1} I_{6} ;{ }^{3} P_{0^{-}}{ }^{3} H_{4}$ [21].

Despite the obvious attractiveness such recombination was not favorable. This is due to two reasons, first of all the first step in the cascade resulted in UV emission. Moreover, we also detected the direct recombination from the ${ }^{1} S_{0}$ state to the ground ${ }^{3} H_{J}$ multilpets. The apparent solution was to block the above-mentioned two transitions. We thus looked for other systems, in which $\mathrm{Pr}^{3+}$ excitation (due to overlapping $4 f^{2}$ to $4 f^{1} 5 d^{1}$, charge transfer and host excitations) is shifted down in energy. This we observed for $\mathrm{ZrO}_{2}$ nanoparticles doped with $\mathrm{Pr}^{3+}$ ions (with $1 \%$ concentration) [16].

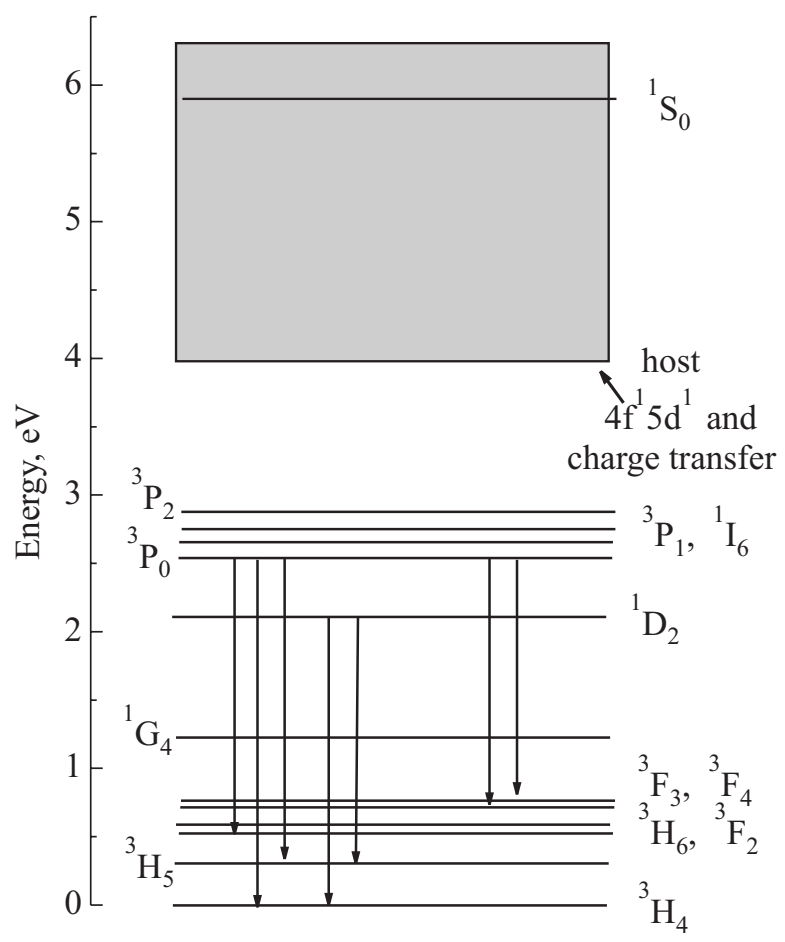

Fig. 6. The energy level scheme of $\mathrm{Pr}^{3+}$ ion in $\mathrm{ZrO}_{2}$ lattice with the indicated scheme of cascade processes following the initial $4 f-5 d$, charge transfer or host excitation.
We reported recently (see [16] for further details) that a broad excitation band in $\mathrm{ZrO}_{2}$ (due to an overlap of $4 f^{2}-4 f^{1} 5 d^{1}$, charge transfer transition and band-to-band host excitation) overlaps with the ${ }^{1} S_{0}$ state. In this case the $\mathrm{Pr}^{3+} \mathrm{PL}$ is dominated by the red ${ }^{1} \mathrm{D}_{2}{ }^{3} \mathrm{H}_{4}$ transition observed together with the green ${ }^{3} \mathrm{P}_{0^{-}}{ }^{3} \mathrm{H}_{4}$ transition. Under $250 \mathrm{~nm}$ excitation $\mathrm{Pr}^{3+} \mathrm{PL}$ is fairly efficient and moreover consists of several overlapping bands covering a whole spectral region of the visible light. An impression of the white light emission results, with good colorimetric parameters of this white emission [28].

\section{Summary and conclusions}

Our extensive investigations led to the conclusion that the intra-shell emission of Mn and RE ions is significantly enhanced in nanocrystals. A clear correlation between size of nanoparticles and PL efficiency is demonstrated. There are several possible explanations of the PL enhancement. For example, the enhanced efficiency of host to $\mathrm{Mn}^{2+}$ energy pumping and also confinement enhanced Mn-free carriers spin-flip interactions (see Refs. 5-12) are responsible for the increased quantum efficiency of the ${ }^{4} T_{1}$ to ${ }^{6} A_{1}$ intra-shell PL in nanocrystals. For RE ions we postulate an important role of super-radiance processes. Photon cascade processes and the way of their optimization are also discussed.

This work was partly supported by grant No 1 P03B 09030 of MEiN, Poland.

1. Energy Savings Potential of Solid State Lighting in General Illumination Applications, Prepared by: Navigant Consulting Inc., $1801 \mathrm{~K}$ Street, NW, Suite 500, Washington, DC 20006.

2. M. Godlewski and A. Kozanecki, Gallium Nitride and Related Materials, in Handbook of Electroluminescent Materials, D.R. Vij (ed.), Institute of Physics Publishing of U.K., Bristol and Philadelphia, IOP Publishing (2004), p. 348 .

3. S. Nakamura, M. Senoh, S. Nagahama, T. Matsishita, H. Kiyoku, Y. Sugimoto, T. Kozaki, H. Umemoto, M. Sano, and T. Mukai, Jpn. J. Appl. Phys. Part 2, 38, L226 (1999).

4. S. Nakamura, M. Senoh, S. Nagahama, N. Iwasa, T. Matsushita, and T. Mukai, Appl. Phys. Lett. 76, 22 (2000).

5. M. Godlewski, V.Yu. Ivanov, A. Khachapuridze, and S. Yatsunenko, Physica Status Solidi (b) 229, 533 (2002).

6. M. Godlewski, S. Yatsunenko, A. Khachapuridze, V.Yu. Ivanov, Z. Gołacki, G. Karczewski, P.J. Bergman, P.J. Klar, W. Heimbrodt, and M.R. Phillips, J. Alloys Compd. 380, 45 (2004).

7. M. Godlewski, S. Yatsunenko, V.Yu. Ivanov, A. Khachapuridze, K. Świątek, E.M. Goldys, M.R. Phillips, P.J. Klar, and W. Heimbrodt, Acta Physica Polonica A107, 65 (2005).

8. M. Godlewski, S. Yatsunenko, K. Drozdowicz-Tomsia, E.M. Goldys, M.R. Phillips, P.J. Klar, and W. Heimbrodt, Acta Physica Polonica A108, 681 (2005). 
9. M. Godlewski, S. Yatsunenko, V.Yu. Ivanov, K. Drozdowich-Tomsia, E.M. Goldys, M.R. Phillips, P.I. Klar, and W. Heimbrodt, Fiz. Nizk. Temp. 33, 263 (2007) [Low Temp. Physics 33, 192 (2007)].

10. M. Godlewski, S. Yatsunenko, and V.Yu. Ivanov, Israeli J. Chem. 46, No. 4, 413 (2006), Special Issue: Magnectic Resonance and Spintronics, Guest Editor: Efrat Lifschitz.

11. M. Godlewski, V.Yu. Ivanov, and S. Yatsunenko, Optical Materials 30, 669 (2008).

12. S. Yatsunenko, M. Godlewski, E. Guziewicz, M. Zalewska, A. Kłonkowski, B. Kukliński, M. Grinberg, P.J. Klar, and W. Heimbrodt, J. Alloys Compd. 451, 206 (2008).

13. M. Godlewski and M. Leskelä, CRC Critical Reviews in Solid State and Materials Sciences 19, 199 (1994).

14. R.N. Bhargava, D. Gallagher, X. Hong, and A. Nurmikko, Phys. Rev. Lett. 72, 416 (1994).

15. L. Chen, F.J. Brieler, M. Fröba, P.J. Klar, and W. Heimbrodt, Phys. Rev. B75, 241303(R) (2007).

16. M. Godlewski, S. Yatsunenko, A. Nadolska, A. Opalińska, W. Łojkowski, K. Drozdowicz-Tomsia, and EM. Goldys, Opt. Mater. (2008), doi: 10.1016/j.optmat.2007.10.020.

17. R.H. Dicke, Phys. Rev. 93, 99 (1954).
18. M. Scheibner, T. Schmidt, L. Worschech, A. Forchel, G. Bacher, T. Passow, and D. Hommel, Nature Physics 3, 106 (2007).

19. S. Yatsunenko et al., unpublished results.

20. E. Wolska et al., unpublished results.

21. W.W. Piper, J.A. DeLuca, and F.S. Ham, J. Lumin. 8, 344 (1973).

22. A.P. Vink, P. Dorenbos, J.T.M. de Haas, H. Donker, P.A. Rodnyi, A.G. Avanesov, and C.W.E. Eijk, J. Phys.: Condens. Matter 14, 8889 (2002).

23. P.A. Rodnyi, A.N. Mishin, and A.S. Potapov, Optics and Spectroscopy 93, 714 (2002).

24. S. Kück, I. Sokólska, M. Henke, M. Döring, and T. Scheffler, J. Lumin. 102-103, 176 (2003).

25. Shi-hua Huang, Xiao-jun Wang, Bao-jiu Chen, D. Jia, and W.M. Yen, J. Lumin. 102-103, 344 (2003).

26. A. Al-Hilfy et al, J. Physics B: Atomic, Molecular, and Optical Physics 18, 3697 (1985).

27. B. Henderson and G.F. Imbusch, in Optical Spectroscopy of Inorganic Solids, Oxford (1989).

28. S. Yatsunenko et al., to be published. 